\title{
Dynamic Response Analysis of Offshore Installation Process for Large Precise Equipment
}

\author{
Ming Zhu ${ }^{\mathrm{a}}$, Peng Zhang ${ }^{\mathrm{b}}$, Changming Zhu ${ }^{\mathrm{c}}$ \\ School of Mechanical engineering, Shanghai Jiaotong University, Shanghai 200240, China \\ azhuming1208@sjtu.edu.cn, bzhp_roc@sjtu.edu.cn, czhuchangming@sjtu.edu.cn
}

Keywords: Dynamic response, large precise equipment, offshore installation, vibration isolator.

\begin{abstract}
A dynamic model of offshore installation process is established for the large precise equipment. To prevent the precise equipment being damaged, vibration isolators are used to absorb the impact energy during the installation process. In this study, the motion of equipment consists of the vertical displacement and rotation based on the large size of equipment. In the model, the tensioning and relaxing of hoisting rope and the constraint from the installation platform are considered. Then, the Baumgartner method is used to modify the dynamic motions. After that, the maximal release velocity is evaluated on the basis of the limit acceleration of equipment according to the motion of winch. Besides, the effect of the different types of damping is investigated by numerical simulation. The results show that vibration isolator with quadratic damping has a better performance and can increase the performance conditions.
\end{abstract}

\section{Introduction}

Recently, there is a strong trend that the offshore installation increases greatly. In order to carry out offshore installation, equipment needs to be transferred from floating crane to installation platform safely. While the severe sea condition increases the pendulum of the equipment and reduces security of installation. Especially, the heave motion of the floating crane has a significant effect on the motion of the equipment during the installation process. In order to ensure safe operation, the peak acceleration of equipment cannot be more than a certain value during the installation process. For safer installation process, it is urgent to study the dynamic response of the equipment during the installation process.

Some works on the offshore suspending process are reported in the literatures. Ellermann et al. [1] developed a nonlinear coupled carne-cargo model based on the assumption that the cargo was a point mass. Ju-Hwan Cha et al. [2] established the coupled model between the floating crane and the cargo considering the geometries of cargo. The current practice is that the heave compensator uses either winch or hydraulic piston to control the payout of the winch line. In principle, the passive heave compensator is adding a spring-damper system to the hoisting rope system studied by Huster et al. [3] and Jia Ni et al. [4]. Lab testing shows a motion reduction of 68\%. Zhang Liangjuan et al. [5] designed a shock isolator with hydraulic damper for heavy structure installation. The hydraulic damper force was optimized and expected to be a constant force. Bo Woo Nam et al. [6, 7] studied the fully coupled dynamics of floating crane vessels, crane wires and lifted equipment. And then, a generalized spring-damper system with a PD feedback controller is used for the deep water installation by simulation and experiments.

In the above study, they mainly focused on the lifting process and the tensioning force of hoisting rope remains the same as far as possible using the heave compensator. But for the specific installation process of large equipment, it is not appropriate to treat the equipment as a point mass compared above models. During the installation process, the motion of equipment is restricted by the installation platform. Therefore, it is necessary to build the dynamic model for the offshore installation process and the dynamic characteristic is studied. In this study, the nonlinear time-domain analysis for offshore installation is carried out. The dynamic model of equipment consists of the tensioning and relaxing of hoisting rope and the displacement constraint from the installation platform. Then, the Lagrange multiplier is introduced to describe the contact element between the equipment and the installation 
platform. Last, the dynamic characteristics of the equipment are analyzed to maintain an acceptable performance during the installation process.

\section{Development of dynamic analysis for installation process}

During the offshore installation process, the horizontal motion of floating crane can be controlled by conventional dynamic positioning system or mechanical positioning as described in the literature [8]. Therefore, the motion of the floating crane tip in the horizontal plane is not considered. The basic model of installation process is shown in the Fig.1. In the Fig.1, the dotted line represents winch, equipment and structural frame of vibration isolator. The body-fixed coordinate system $\mathrm{O}_{1} \mathrm{Z}_{1}$ is defined as being put to the point $A$ of the equipment at the initial time. $z_{1}$ and $\theta$ represent the position and rotation angle of the equipment, respectively. The coordinate system $\mathrm{O}_{2} \mathrm{Z}_{2}$ is defined as being put to the plunger at the initial time. $z_{2}$ and $z_{3}$ are the position of the plungers of the two vibration isolators, respectively.

Fig. 1 gives the height $H$ and width $L$ of equipment and the distance $R$ between the suspending point A and the center of mass. The hoisting rope is modeled as a generalized spring-damper system with a spring $K_{r}$ and a damper $C_{r}$. And then, two vibration isolators which can be simplified as stiffness-damping elements are mounted at point $\mathrm{B}$ and point $\mathrm{C}$ to mitigate the shock energy during the installation process. At the initial time, the equipment is supposed to be located right above the installation platform. The initial release height is $H_{0}$ and the maximum stroke of vibration isolator is $S$.

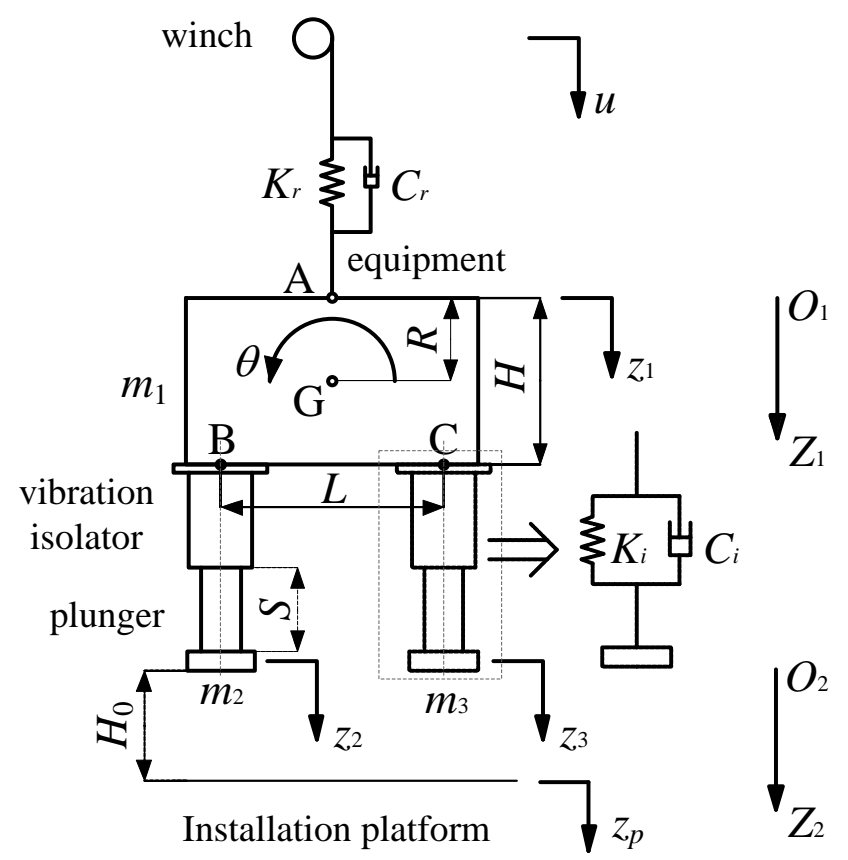

\subsection{Winch's motion.}

Fig.1 Diagram of installation model system

The winch is assumed to be located at the floating crane tip. The heave motion of floating crane tip is combined with a variety of sinusoidal waves: amplitudes and periods [8]. The sinusoidal waves usually range the amplitude of few meters with the wave frequencies of $0.05-0.2 \mathrm{~Hz}$. Here, the typical amplitude and frequency of wave is chosen for the simulation as follows.

$$
z_{f}(t)=A \sin (2 \pi f t+\varphi)
$$

where the positive constants $A, f$ and $\varphi$ denote the amplitude, frequency and phase of floating crane tip, respectively. Therefore, the motion of winch considering the release velocity can be expressed by,

$$
u(t)=z_{f}(t)+v_{0} t
$$

where $v_{0}$ is the release velocity of equipment. 


\subsection{Dynamic model of the load motion without constraints.}

Depending on the position of the equipment, the installation process mainly includes two processes: one is the suspending process and the other is the landing process which at least one of the vibration isolators land on installation platform. The schematic diagram of installation process is given in Fig.2.

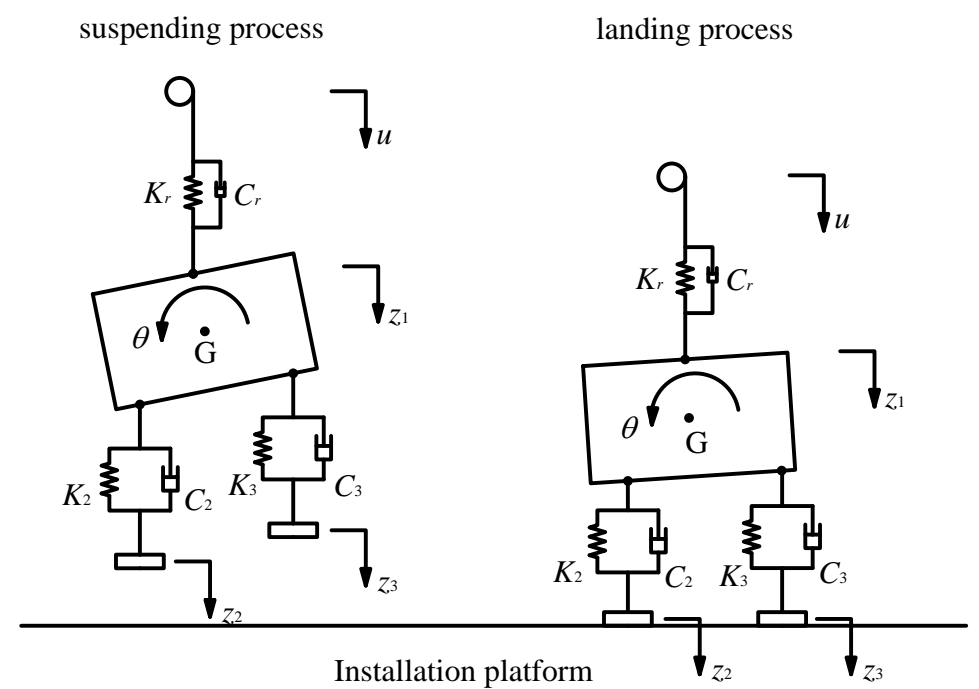

Fig.2 Diagram of installation process

In the actual condition, the plunger mass $m_{2}$ and $m_{3}$ are much less than the equipment mass $m_{1}$. Therefore, the plunger mass do not affect the center of mass of equipment during the whole installation process.

According to the Lagrange equation, the dynamic motion of equipment during the suspending process can be obtained considering the small rotation angle,

$$
\mathbf{M Z}=\mathbf{F}
$$

where

$$
\mathbf{M}=\left[\begin{array}{cccc}
m_{1} & 0 & 0 & 0 \\
0 & I_{G}+m_{1} R^{2} & 0 & 0 \\
1 & L / 2 & -1 & 0 \\
1 & -L / 2 & -1 & 0
\end{array}\right], \quad \mathbf{Z}=\left[\begin{array}{c}
z_{1} \\
\theta \\
z_{2} \\
z_{3}
\end{array}\right], \quad \mathbf{F}=\left[\begin{array}{c}
m_{1} g+m_{1} R \dot{\theta}^{2}-F_{r} \\
-m_{1} R \dot{\theta} \dot{z}_{1}-F_{r} R \theta \\
0 \\
0
\end{array}\right]
$$

where $I_{G}$ is the moment of inertia of the center of mass of equipment. $F_{r}$ represents the tensioning force of hoisting rope.

\subsection{Constraint analysis.}

The constraints applied at the equipment should be considered during the landing process. These constraints are defined as follows,

(1) The tensioning force of the hoisting rope. During the landing process, the equipment is released down by the hoisting rope. Due to the heave motion of floating crane, the hoisting rope may be relaxed and tensioned depending on the distance between the winch and suspending point $\mathrm{A}$.

The overall length of hoisting rope $l_{R}$ can be written as follows,

$$
l_{R}(t)=l_{R}\left(t_{0}\right)+v_{0}(t)+r(t)
$$

where $l_{R}\left(t_{0}\right)$ denotes the rope length at the initial time $t_{0} . r(t)$ is the elastic extension of hoisting rope. The spring stiffness of hoisting rope can be expressed as follows,

$$
K_{r}=\frac{n_{r} E_{r} A_{r}}{l_{R}(t)}
$$

where $E_{r}$ and $A_{r}$ denote Young's modulus and the intersectional area of the rope, respectively. $n_{r}$ is the number of rope.

Therefore, the tensioning force of hoisting rope is given in Eq. (7). 


$$
F_{r}=\left\{\begin{array}{cc}
K_{r}\left(r+z_{1}-u\right)+C_{r}\left(\dot{r}+\dot{z}_{1}-\dot{u}\right) & r+z_{1}-u \geq 0 \\
0 & r+z_{1}-u<0
\end{array}\right.
$$

(2) When the plunger contacts with the installation platform, displacement constraint equations from the installation platform should be considered,

$$
\begin{array}{ll}
\Phi_{2}=z_{2}-z_{p}=z_{c_{1}} & z_{2}-z_{p} \geq z_{c_{1}} \\
\Phi_{3}=z_{3}-z_{p}=z_{c_{1}} & z_{3}-z_{p} \geq z_{c_{1}}
\end{array}
$$

where $z_{c_{1}}$ is the initial position of the installation platform in the coordinate system $\mathrm{O}_{2} \mathrm{Z}_{2} . z_{p}$ is the heave motion of the installation platform. In the actual condition, the installation platform is fixed on the sea bed by the pile pipes. Therefore, the installation platform can be considered to be static. That means, $z_{p}=0$.

(3) During the landing process, the force produced by the vibration isolator is applied to the equipment.

$$
\begin{aligned}
& F_{2}=\left\{\begin{array}{cc}
f\left(K_{2}, C_{2}, z_{B}, \dot{z}_{B}, z_{2}, \dot{z}_{2}\right) & z_{B} \geq z_{2} \\
0 & z_{B}<z_{2}
\end{array}\right. \\
& F_{3}=\left\{\begin{array}{cc}
f\left(K_{3}, C_{3}, z_{C}, \dot{z}_{C}, z_{3}, \dot{z}_{3}\right) & z_{C} \geq z_{3} \\
0 & z_{C}<z_{3}
\end{array}\right.
\end{aligned}
$$

where $K_{i}$ and $C_{i}(i=2,3)$ represent the stiffness and damping of the vibration isolators, respectively. $F_{2}$ and $F_{3}$ represent the forces produced by the vibration isolators.

(4) During the landing process, the actual vibration isolator has stroke limitation. Here, the stroke constraint equations of vibration isolator should be introduced into the motion equations of equipment when vibration isolator completes its stroke.

$$
\begin{array}{ll}
\Phi_{4}=z_{B}-z_{p}=z_{c_{2}} & z_{B}-z_{p} \geq z_{c_{2}} \\
\Phi_{5}=z_{C}-z_{p}=z_{c_{2}} & z_{C}-z_{p} \geq z_{c_{2}}
\end{array}
$$

Where $z_{c_{2}}$ is the position of the installation platform in the coordinate system $\mathrm{O}_{1} \mathrm{Z}_{1}$.

\subsection{Dynamic model of the equipment with constraints.}

According to the above analysis, the displacement constraint equation and constraint force equation can be obtained.

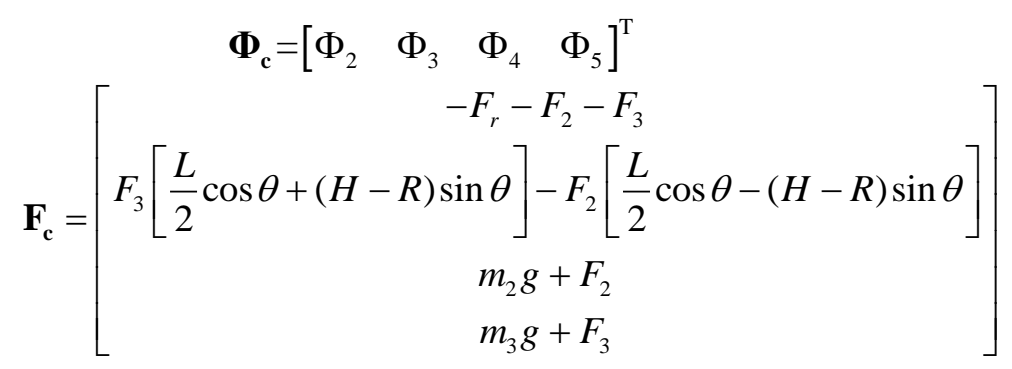

During the installation process, the above constraint equations change all the time depending on the position of the equipment. Therefore, the constraint equations need to be updated at each iteration step during the simulation.

Then, Eq. (14) can be transformed to the following equation,

$$
\ddot{\Phi}_{c}=\Phi_{J} \ddot{\mathbf{Z}}-\gamma
$$

The matrix $\Phi_{\mathrm{J}}$ is defined as the Jacobian matrix of $\boldsymbol{\Phi}_{\mathrm{c}}$.

$$
\boldsymbol{\Phi}_{\mathbf{J}}=\left[\begin{array}{cccc}
0 & 0 & 1 & 0 \\
0 & 0 & 0 & 1 \\
1 & L / 2 & -1 & 0 \\
1 & -L / 2 & -1 & 0
\end{array}\right] \quad \gamma=\left[\begin{array}{llll}
\ddot{z}_{c_{1}} & \ddot{z}_{c_{1}} & \ddot{z}_{c_{2}} & \ddot{z}_{c_{2}}
\end{array}\right]^{\mathrm{T}}
$$

The equipment motion can be set up including the constraint equations, 


$$
\left[\begin{array}{cc}
\mathbf{M} & \Phi_{\mathrm{J}}^{\mathrm{T}} \\
\Phi_{\mathrm{J}} & 0
\end{array}\right]\left[\begin{array}{l}
\ddot{\mathbf{Z}} \\
\lambda
\end{array}\right]=\left[\begin{array}{c}
\mathbf{F}+\mathbf{F}_{\mathbf{c}} \\
\gamma
\end{array}\right]
$$

where the Lagrange multiplier $\lambda$ is used to describe the contact force between the equipment and the installation platform.

Then, the Baumgarte method $[9,10,11]$ is used to modify the dynamic motions as shown in Eq. (19).

$$
\left[\begin{array}{cc}
\mathbf{M} & \boldsymbol{\Phi}_{\mathbf{J}}^{\mathbf{T}} \\
\boldsymbol{\Phi}_{\mathbf{J}} & 0
\end{array}\right]\left[\begin{array}{l}
\ddot{\mathbf{Z}} \\
\boldsymbol{\lambda}
\end{array}\right]=\left[\begin{array}{c}
\mathbf{F}+\mathbf{F}_{\mathbf{c}} \\
\gamma-2 \alpha_{B} \dot{\boldsymbol{\Phi}}_{\mathbf{c}}-\beta_{B}^{2} \boldsymbol{\Phi}_{\mathbf{c}}
\end{array}\right]
$$

where $\alpha_{B}$ and $\beta_{B}$ are Baumgarte coefficients. Here, the coefficients $\alpha_{B}$ and $\beta_{B}$ can be calculated as follows [12],

$$
\alpha_{B}=\left\{\begin{array}{lc}
100 e_{n} & e_{2} \geq-6 \\
0 & e_{2} \leq-16 \\
10\left(16+e_{2}\right) e_{n} & -16<e_{2}<-6
\end{array} \quad, \quad \beta_{B}=\left\{\begin{array}{lc}
100 e_{n} & e_{1} \geq-6 \\
0 & e_{1} \leq-16 \\
10\left(16+e_{1}\right) e_{n} & -16<e_{1}<-6
\end{array}\right.\right.
$$

$e_{1}$ and $e_{2}$ can be expressed as,

$$
e_{1}=\lg \sqrt{\frac{\Phi_{\mathbf{c}}^{\mathrm{T}} \Phi_{\mathbf{c}}}{n}}, \quad e_{2}=\lg \sqrt{\frac{\dot{\Phi}_{\mathbf{c}}^{\mathrm{T}} \dot{\Phi}_{\mathbf{c}}}{n}}
$$

where $n$ is the number of the constraint equations and $e_{n}$ is the calculation error at time $t_{n}$.

\section{Configuration of the dynamic response simulation}

The configuration of the dynamic response simulation in this paper is shown in Fig. 3. First, the 2-DOF plane motion equations of equipment are derived. Second, the displacement constraint equation and constraint force equation are determined according to the solutions from the previous iteration. Then, the motion equations of equipment including the above constraint equations can be obtained. Finally, the dynamic motions are solved by the Baumgarte method until the installation process is completely finished.

The general operation and main properties parameters of the system are shown in Table 1.

Table 1. Parameters

\begin{tabular}{cc}
\hline Parameters & Value \\
\hline Initial release height $H_{0}(\mathrm{~m})$ & 1 \\
Shock isolation stroke $S(\mathrm{~m})$ & 1 \\
Plunger mass $m_{2}(\mathrm{~kg})$ & $10 \times 10^{3}$ \\
Plunger mass $m_{3}(\mathrm{~kg})$ & $10 \times 10^{3}$ \\
Equipment mass $m_{1}(\mathrm{~kg})$ & $360 \times 10^{3}$ \\
Distance of center of gravity $R(\mathrm{~m})$ & 25 \\
Initial rope length $l_{R 0}(\mathrm{~m})$ & 20 \\
Rope area $A_{r}\left(\mathrm{~m}^{2}\right)$ & $1.77 \times 10^{-4}$ \\
Initial rotation angle $\theta$ & 0.011 \\
\hline
\end{tabular}




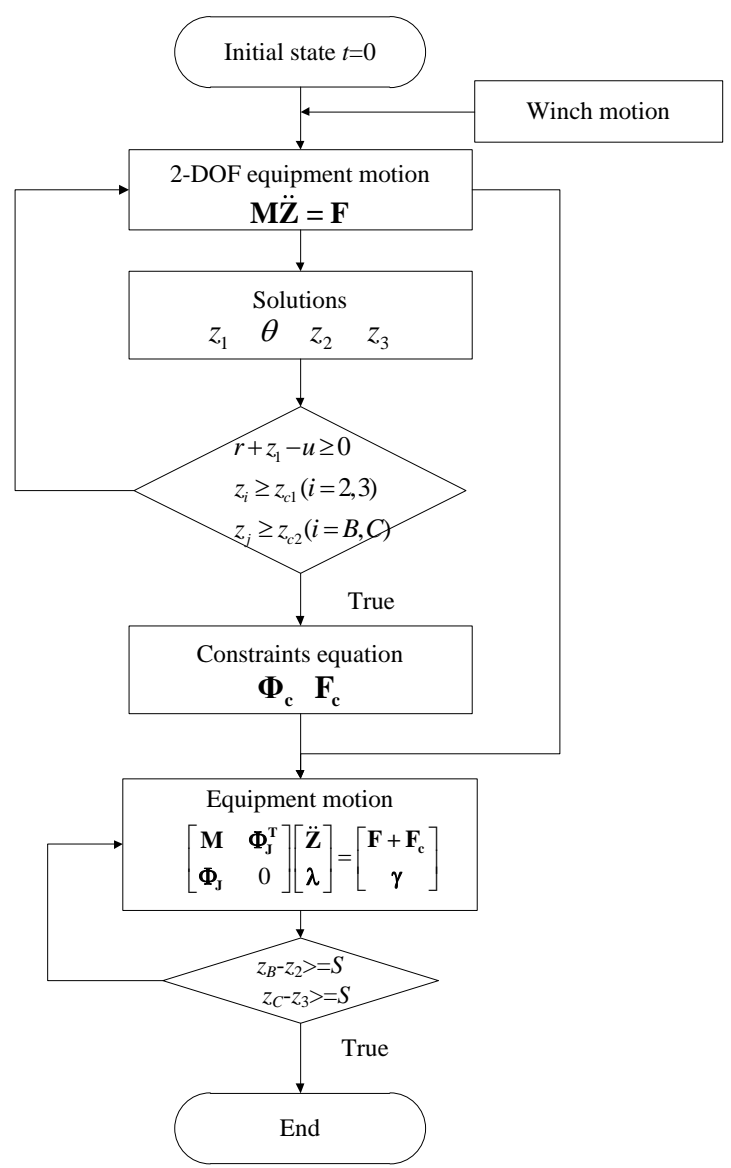

Fig.3 Configuration of the dynamic response simulation

\section{Releasing velocity evaluation}

First, the equipment is supposed to be released close to the installation platform. Thus, the stiffness of hoisting rope is little affected by the length of rope. The stiffness $K_{r}$ can be assumed as a constant. As a result, the extension of hoisting rope $r$ can also be considered as a constant. Furthermore, the motion of equipment during the suspending process can be simplified when the rotation of equipment is very small.

$$
\left(m_{1}+m_{2}+m_{3}\right) \ddot{z}_{1}=\left(m_{1}+m_{2}+m_{3}\right) g-K_{r}\left(r+z_{1}-u\right)-C_{r}\left(\dot{r}+\dot{z}_{1}-\dot{u}\right)
$$

Here, $\dot{r}=0$ and the coefficients $A_{1}=\frac{K_{r}}{\left(m_{1}+m_{2}+m_{3}\right)} A, A_{2}=\frac{C_{r}}{\left(m_{1}+m_{2}+m_{3}\right)} A \omega, A_{3}=\frac{C_{r}}{\left(m_{1}+m_{2}+m_{3}\right)}$, $A_{4}=\frac{K_{r}}{\left(m_{1}+m_{2}+m_{3}\right)}$ are introduced into the equation. As a result, the motion of equipment can be rewritten as,

$$
\ddot{z}_{1}+A_{3} \dot{z}_{1}+A_{4} z_{1}=A_{1} \sin (\omega t)+A_{2} \cos (\omega t)+A_{3} v_{0}+A_{4} v_{0} t
$$

where $\omega=2 \pi f$ and the phase angle $\varphi=0$.

Therefore, the solution can be obtained as,

$$
z_{1}=e^{\alpha t}\left(c c_{1} \cos \beta t+c c_{2} \sin \beta t\right)+B_{1} \sin \omega t+B_{2} \cos \omega t+B_{3} t
$$

where

$$
B_{1}=\frac{A_{1}\left(-\omega^{2}+A_{4}\right)+A_{2} A_{3} \omega}{\left(-\omega^{2}+A_{4}\right)^{2}+\left(A_{3} \omega\right)^{2}}, \quad B_{2}=\frac{A_{2}\left(-\omega^{2}+A_{4}\right)-A_{1} A_{3} \omega}{\left(-\omega^{2}+A_{4}\right)^{2}+\left(A_{3} \omega\right)^{2}}, \quad B_{3}=v_{0}, \alpha=-\frac{A_{3}}{2}, \beta=\sqrt{4 A_{4}-A_{3}^{2}}
$$

The following initial conditions are considered,

$$
z_{1}(0)=0, \quad \dot{z}_{1}(0)=v_{0}
$$


Then, the coefficients $c c_{1}$ and $c c_{2}$ can be obtained.

$$
c c_{1}=-B_{2}, \quad c c_{2}=-\frac{\alpha c c_{1}+B_{1} \omega+B_{3}}{\beta}
$$

The acceleration of equipment can be given by,

$$
\begin{aligned}
& \ddot{z}_{1}=e^{\alpha t}\left[\left(\alpha^{2}-\beta^{2}\right) c c_{1}+2 \alpha \beta c c_{2}\right] \cos \beta t+e^{\alpha t}\left[\left(\alpha^{2}-\beta^{2}\right) c c_{2}-2 \alpha \beta c c_{1}\right] \sin \beta t \\
& -\left(B_{1} \omega^{2} \sin \omega t+B_{2} \omega^{2} \cos \omega t\right)
\end{aligned}
$$

Thus, the peak acceleration can be obtained,

$$
\left(\ddot{z}_{1}\right)_{\min }=-e^{\frac{3 \alpha \pi}{2 \beta}}\left[\left(\alpha^{2}-\beta^{2}\right) c c_{2}-2 \alpha \beta c c_{1}\right]
$$

The maximum release velocity can be obtained as,

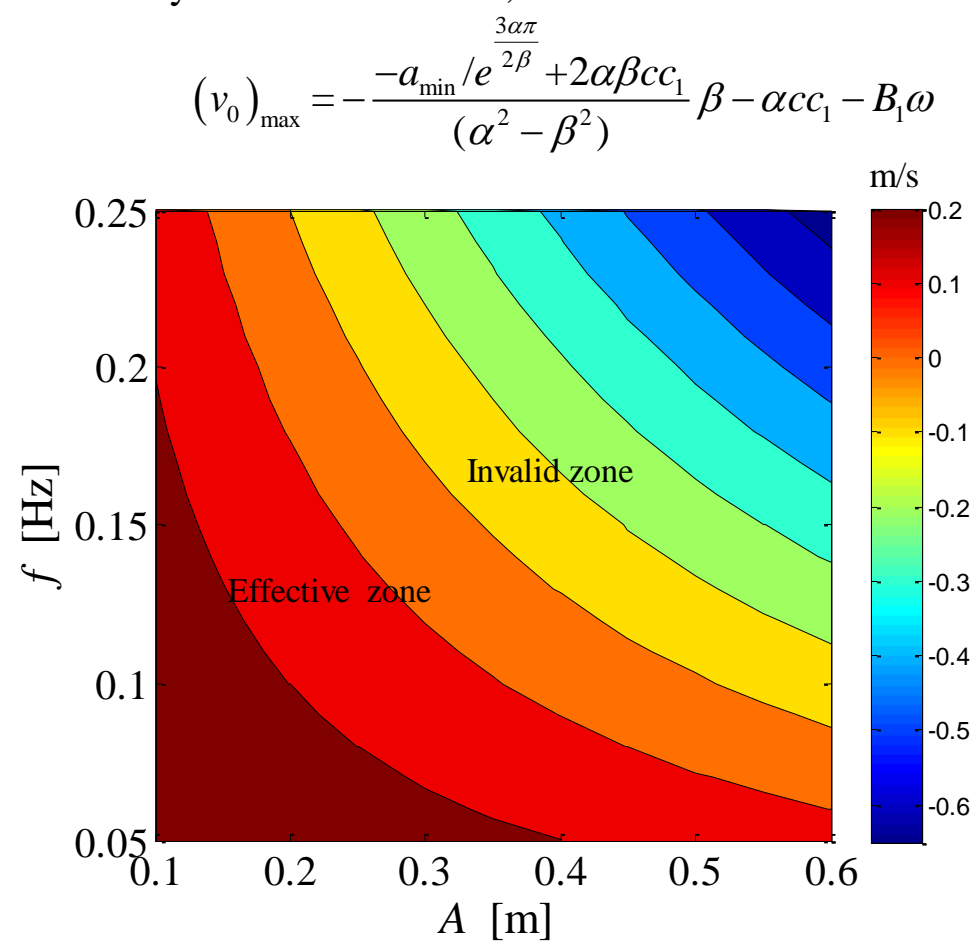

Fig.4 Contour plot of release velocity

In the actual condition, the peak acceleration of equipment is expected to be less than $0.5 \mathrm{~g}$. That is to say, $a_{\min }=-0.5 \mathrm{~g}$. Fig. 4 gives the contour plot of maximum release velocity. From the results, it can be found that the maximum release velocity is $0.2 \mathrm{~m} / \mathrm{s}$ when the winch motion has lower frequency and smaller amplitude. While, when the winch motion has higher frequency or larger amplitude, it is not suitable for the offshore installation.

\section{Results and analysis}

According to the Fig.4, the maximum release velocity is $9.17 \mathrm{~m} / \mathrm{min}$ when the frequency, amplitude and phase are $0.1 \mathrm{~Hz}, 0.3 \mathrm{~m}$ and 0 , respectively. For the safety's sake, the release velocity is set as 4 $\mathrm{m} / \mathrm{min}$. Here, two types of the typical damping in the hydraulic vibration isolator are used to reduce the peak acceleration during the landing process. One is the linear vibration isolator with linear stiffness and linear damping. The other one is the nonlinear vibration isolator with linear stiffness and quadratic damping. The forces produced by the vibration isolators can be expressed as,

For the linear vibration isolator, 


$$
\begin{aligned}
& F_{2}=\left\{\begin{array}{cc}
K_{2}\left(z_{B}-z_{2}\right)+C_{2}\left(\dot{z}_{B}-\dot{z}_{2}\right) & z_{B} \geq z_{2} \\
0 & z_{B}<z_{2}
\end{array}\right. \\
& F_{3}=\left\{\begin{array}{cc}
K_{3}\left(z_{C}-z_{3}\right)+C_{3}\left(\dot{z}_{C}-\dot{z}_{3}\right) & z_{C} \geq z_{3} \\
0 & z_{C}<z_{3}
\end{array}\right.
\end{aligned}
$$

For the nonlinear vibration isolator,

$$
\begin{aligned}
& F_{2}=\left\{\begin{array}{cc}
K_{2}\left(z_{B}-z_{2}\right)+C_{2}\left(\dot{z}_{B}-\dot{z}_{2}\right)\left|\dot{z}_{B}-\dot{z}_{2}\right| & z_{B} \geq z_{2} \\
0 & z_{B}<z_{2}
\end{array}\right. \\
& F_{3}=\left\{\begin{array}{cc}
K_{3}\left(z_{C}-z_{3}\right)+C_{3}\left(\dot{z}_{C}-\dot{z}_{3}\right)\left|\dot{z}_{C}-\dot{z}_{3}\right| & z_{C} \geq z_{3} \\
0 & z_{C}<z_{3}
\end{array}\right.
\end{aligned}
$$

The stiffness of vibration isolator are set as $K_{i}=2 \times 10^{6} \mathrm{~N} / \mathrm{m}$ and $K_{i}=2 \times 10^{5} \mathrm{~N} / \mathrm{m}$, respectively. The damping of vibration isolator are $C_{i}=5 \times 10^{6}(i=2,3)$. The displacement, acceleration of center of mass and tensioning force are shown as in the Fig.5.
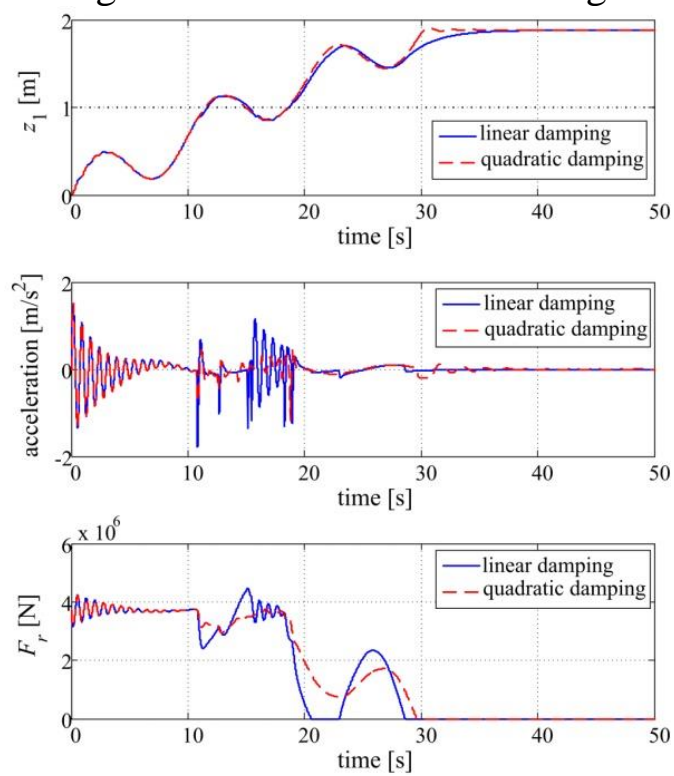

(a) $K_{i}=2 \times 10^{6} \mathrm{~N} / \mathrm{m}$
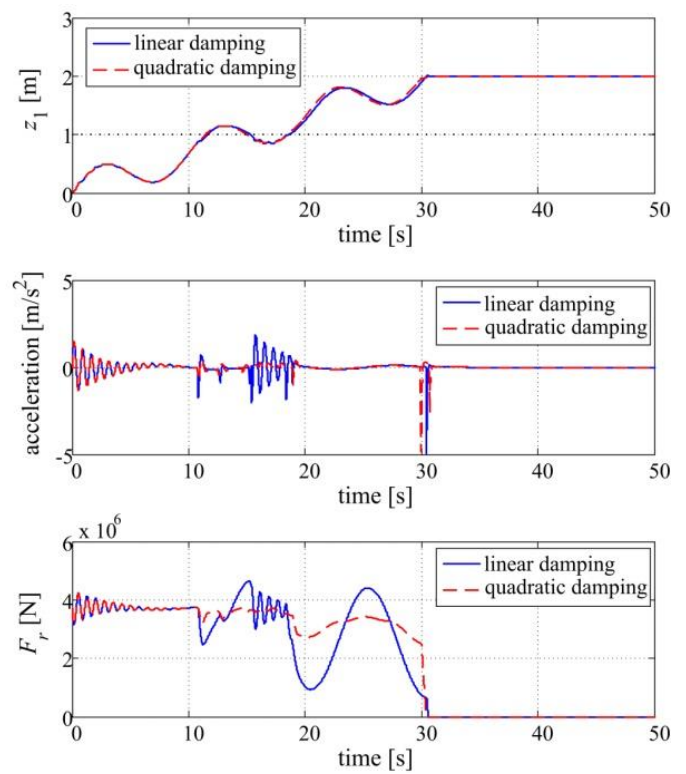

(b) $K_{i}=2 \times 10^{5} \mathrm{~N} / \mathrm{m}$

Fig. 5 Dynamic response of the installation process

When the stiffness of vibration isolator $K_{i}$ is $2 \times 10^{6} \mathrm{~N} / \mathrm{m}$, the equipment has two landing times before the equipment is installed completely. From the acceleration curves, it can be found that the peak acceleration of the equipment is $-1.35 \mathrm{~m} / \mathrm{s}^{2}$ and $-1.78 \mathrm{~m} / \mathrm{s}^{2}$. Therefore, the equipment has smaller peak acceleration when the vibration isolator with quadratic damping is used. Furthermore, the acceleration of equipment fluctuates in the tiny range during the landing process. The hoisting rope still has the tensioning force during the landing process and the tensioning force decreases to zero gradually. While, the hoisting rope can be relaxed and tensioned again using the linear damping during the landing process.

When the stiffness of vibration isolator $K_{i}$ is $2 \times 10^{5} \mathrm{~N} / \mathrm{m}$, the equipment also has two landing times before the equipment is installed completely. From the acceleration curves, it can be found that the peak acceleration of the equipment is $-4.94 \mathrm{~m} / \mathrm{s}^{2}$ and $-4.81 \mathrm{~m} / \mathrm{s}^{2}$, which occurs at the final moment of the landing process. That means the equipment still has the impact velocity at the end of landing process. From the above, the larger stiffness of vibration isolator should be chosen for the offshore installation.

Then, the comparison of different damping of vibration isolators is performed in the section. The linear stiffness is $2 \times 10^{6} \mathrm{~N} / \mathrm{m}$. The peak acceleration of center of mass is given in the Fig.6. With the increase of the damping, the peak acceleration of equipment decreases and then increases. It can be 
found that the quadratic damping is more effective than the linear damping except certain damping values.

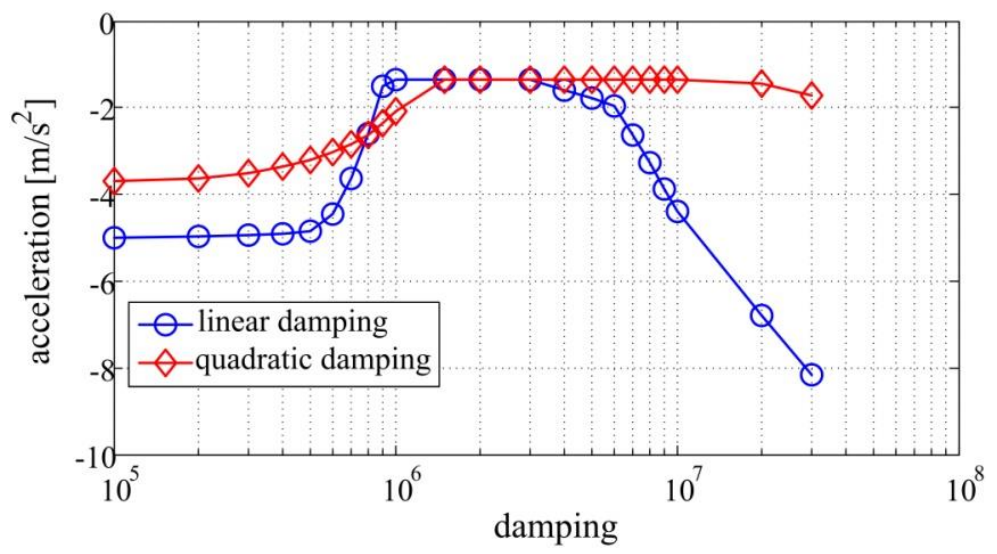

Fig.6 Effect of the damping of vibration isolator

Then, the optimal operation condition can be determined based on the limit acceleration of equipment. The release velocity of equipment is set to $4 \mathrm{~m} / \mathrm{min}$ according to the actual operation condition. Fig.7 shows the contour plot of peak acceleration of equipment. The results show that the vibration isolator with quadratic damping is more effective especially when the motion of winch has larger amplitude and higher frequency.

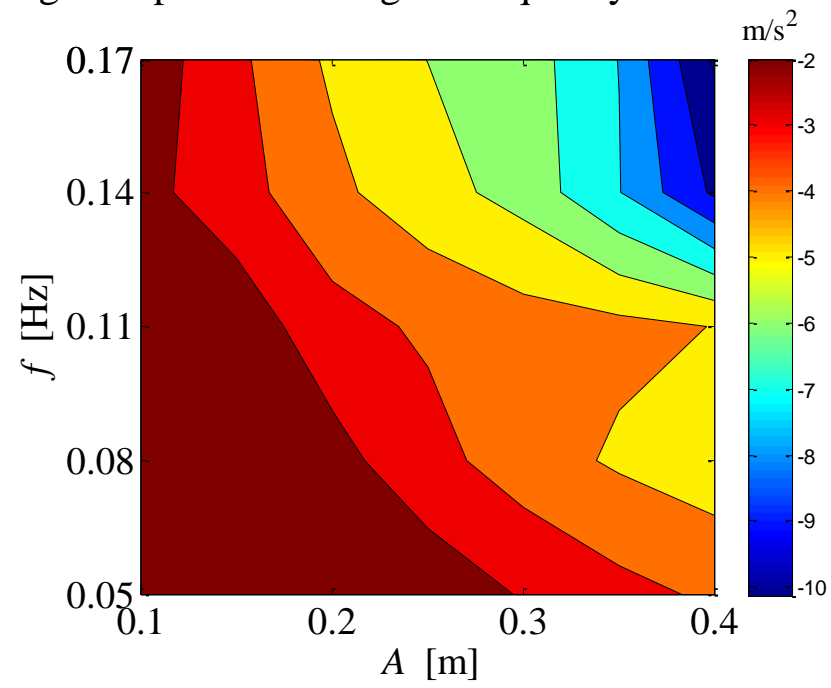

(a) Linear damping

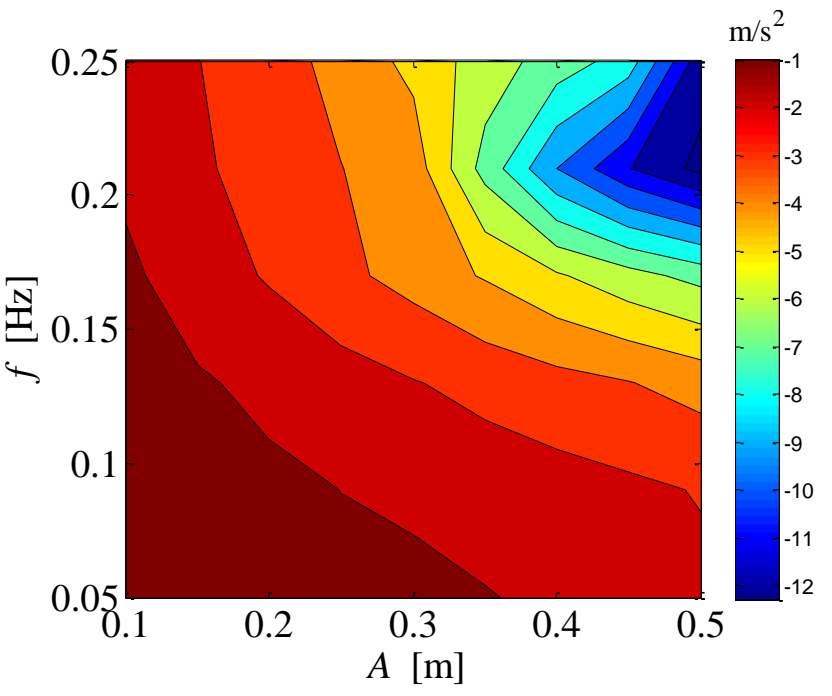

(b) Quadratic damping

Fig.7 Contour plot of equipment acceleration

\section{Summary}

This paper presents an offshore installation dynamic model of large equipment. In the model, it includes the displacement constraint from installation platform and vibration isolator stroke. Furthermore, the tensioning and relaxing of hoisting rope are considered in the model. And then, the Lagrange multiplier is introduced to describe the contact element between the equipment and the installation platform. The model is carried out by the Baumgarte method. Then, the maximum release velocity during the suspending process is estimated according to the heave motion. Last, two vibration isolators with different damping structures are chosen to analyze the characteristics. Through simulation results, the vibration isolator with quadratic damping can work better than the linear damping except the certain damping values and can increase the operating conditions. This model can provide the dynamic analysis method for the offshore installation process and give the advice for designing the vibration isolator for large equipment installation. 


\section{References}

[1]. Ellermann, K., Kreuzer, E., Markiewicz, M. Nonlinear dynamics of floating cranes. Journal of Nonlinear dynamics. Vol.27 (2002) No.2, p. 107-183.

[2]. Ju-Hwan Cha, Myung-ll Roh, et, al. Dynamic response simulation of a heavy cargo suspended by a floating crane based on multibody system dynamics. Journal of Ocean Engineering. Vol. 37 (2010) No.14, p.1273-1291.

[3]. Huster A, Bergstrom H, Gosior J, et al. Design and operational performance of a standalone passive heave compensation system for a work class ROV. Oceans 2009, MTS/IEEE Biloxi-Marine Technology for Our Future: Global and Local Challenges.USA, 2009, p.1-8.

[4]. Ni J, Liu S, Wang M, et al. The simulation research on passive heave compensation system for deep sea mining. Mechatronics and Automation 2009, ICMA 2009. International Conference on. China, 2009, p. 5111-5116.

[5]. Zhang Liangjuan, Zhu Changming, et, al. A novel shock isolator for heavy structure installation. Proceedings of the Institution of Mechanical Engineers, Part C: Journal of Mechanical Engineering Science. Vol. 224 (2010) No.11, p. 283-292.

[6]. Bo Woo Nam, Sa Young Hong, Young Shik Kim. Effects of passive and active heave compensators on deepwater lifting operation. International Journal of offshore and polar engineering. Vol. 23 (2013) No.1, p.33-37.

[7]. Bo Woo Nam, Sa Young Hong, Young Shik Kim et al. An experimental study on deepwater crane installation of subsea equipment in waves. The Twenty-fifth International Ocean and Polar Engineering Conference. USA, 2015, p. 1279-1283.

[8]. Sungil Shin, Jong Kun Lee and Sang Jun Kim. The design of hydraulic simulating apparatus for active heave compensation systems. The Twenty-fourth International Ocean and Polar Engineering Conference. Korea, 2014, p. 919-923.

[9]. Baumgarte J. W. A new method of stabilization for holonomic constraints. Journal of applied mechanics. Vol. 50 (1983) No.4a, p. 869-870.

[10]. Baumgarte J. W. Stabilization of constraints and integrals of motion in dynamical systems. Journal of Computer methods in applied mechanics and engineering. Vol.1 (1972) No.1, p.1-16.

[11]. Hong Zhenjia. Computational multibody dynamics. Higher education press, 1999, p.309-312.

[12]. Liu Ying, Ma Jianmin. Adaptive feedback parameters for Baumgarte's constraint violation stabilization methods of multibody system's equations of motion. Journal of Fudan University. Vol. 51 (2012) No.4, p. 432-436. 